1 Fundação Oswaldo Cruz (Fiocruz), Escola Nacional de Saúde Pública Sérgio Arouca (Ensp) - Rio de Janeiro, Brasil. monique.padilha@hotmail. com

2 Fundação Oswaldo Cruz (Fiocruz), Escola Nacional de Saúde Pública Sérgio Arouca (Ensp)

- Rio de Janeiro, Brasil. Universidade de São Paulo (USP) - São Paulo, Brasil. catia@ensp.fiocruz.br

3 Fundação Oswaldo Cruz (Fiocruz), Escola Naciona de Saúde Pública Sérgio Arouca (Ensp) - Rio de Janeiro, Brasil. anaclaudiafigueiro@gmail. com

\section{Estudo de avaliabilidade do Programa Academia Carioca da Saúde: desafios para a promoção da saúde}

\author{
Evaluability study of the Carioca Health Academy Program: \\ challenges for health promotion
}

Monique Alves Padilha1, Cátia Martins de Oliveira², Ana Cláudia Figueiró

\begin{abstract}
RESUMO Este artigo tem como objetivo apresentar os resultados do estudo de avaliabilidade do Programa Academia Carioca da Saúde visando ampliar a compreensão sobre a intervenção e maximizar a utilidade da avaliação. Foram realizadas as seguintes etapas: análise documental, entrevista com informantes-chave, modelização da intervenção e formulação das perguntas avaliativas. A construção do Modelo Lógico do Programa permitiu descrever as dimensões e os componentes do programa, as ações estratégicas e os efeitos esperados com ênfase no acesso regular às praticas corporais e de atividade física, nos grupos de promoção da saúde e no fortalecimento da participação comunitária.
\end{abstract}

PALAVRAS-CHAVE Avaliação de programas; Promoção da saúde; Planos e programas de saúde.

ABSTRACT This article aims to present the results of the evaluability study of the Carioca Health Academy Program in order to expand the comprehension about the intervention and to maximize the utility of the evaluation. The study was divided into the following steps: documental analyses, interviews with key informants, intervention modeling and elaboration of the evaluative questions. The construction of the Logic Model allowed to describe the dimensions and components of the program, the strategic actions and expected results with emphasis on the regular access to body practices and physical activities, on the health promotion groups and on the strengthening of community participation.

KEYWORDS Program evaluation; Health promotion; Health programs and plans. 


\section{Introdução}

Atualmente, a maioria dos países em desenvolvimento está passando por importantes mudanças em seu perfil demográfico e epidemiológico, reflexo das transformações sociais e econômicas que vêm ocorrendo desde o último século. Se por um lado ainda persistem as doenças transmissíveis, por outro há uma forte carga de Doenças Crônicas Não Transmissíveis (DCNT), constituindo um dos grandes problemas de saúde pública na sociedade moderna (SCHMIDT ET AL., 2011).

Em 2008, cerca de 63\% das mortes por todas as causas no mundo foram atribuídas às DCNT, de acordo com dados da Organização Mundial da Saúde (OMS), sendo que uma parcela expressiva ocorreu principalmente nos países de baixa ou média renda. Dentre os dez principais fatores de risco para as DCNT, o sedentarismo está em quarto lugar, atrás do tabagismo, da alimentação inadequada e do uso prejudicial de álcool, o que indica a importância do fortalecimento de políticas públicas com foco na promoção da saúde e prevenção de doenças (SCHMIDT ET AL., 2011; WHO, 2009). No Brasil essa situação é bastante preocupante, tendo em vista que em 2009 as DCNT representaram $72 \%$ da mortalidade geral e foram responsáveis nos últimos anos por cerca de $69 \%$ dos gastos com internações, gerando um alto custo para o sistema de saúde (FRAGA ET AL., 2013; RIBEIRO; COTTA; RIBEIRO, 2012; BRASIL, 2011B).

Os padrões de atividade física começaram a ser estudados no Brasil mais recentemente e, desde 2006, sua prática tem sido monitorada pelo Vigitel, pesquisa por telefone realizada anualmente pelo Ministério da Saúde (SCHMIDT ET AL., 2011). Dados produzidos por esse estudo indicaram que, em 2009, 16,4\% dos adultos brasileiros eram sedentários não faziam atividade física no tempo livre, durante deslocamentos ou em tarefas como limpeza da casa e trabalho pesado.
Dentre os múltiplos efeitos benéficos proporcionados pela atividade física regular, destacam-se: redução da gordura corporal em razão de um maior gasto calórico, aumento da sensibilidade das células à insulina, diminuição dos níveis de pressão sistólica e diastólica e fortalecimento do sistema imunológico (BRASIL, 2011B). A Organização Mundial da Saúde (OMS) recomenda a prática de 30 minutos de atividade física de intensidade moderada, em cinco ou mais dias por semana.

Em decorrência do alcance global do sedentarismo, principalmente pelas consequências não só para a área de saúde, mas também para os setores econômicos, ambientais, culturais e sociais, o Ministério da Saúde elegeu essa área temática como prioritária, destacando a importância de uma atuação de forma interdisciplinar e intersetorial (KOHL, 2012).

A fim de investir no incentivo a práticas de atividade física, o Brasil tem conduzido diversas iniciativas guiado pelo principal marco de referência da promoção da saúde, a Carta de Ottawa, e pelos princípios e diretrizes do Sistema Único de Saúde discutidos no mesmo ano na VIII Conferência Nacional de Saúde. Cerca de 10 anos depois, foi lançada uma das propostas pioneiras nessa direção denominada Programa Agita São Paulo tendo como objetivo aumentar a prática de atividade física e o conhecimento sobre ações de promoção da saúde pela população do Estado de São Paulo (HALLAL, 2009). Esse programa, apesar de reconhecido pela OMS como modelo para promoção da saúde, sofre alguns questionamentos relacionados a uma aparente culpabilização do sujeito ao determinar padrões de comportamento, entretanto não cabe neste estudo abordar detalhadamente essa questão (FERREIRA; CASTIEL; CARDOSO, 2011).

Posteriormente, outros projetos foram surgindo com foco para além da prática de atividade física, ou seja, envolvendo outros hábitos e comportamentos de vida saudáveis. Na cidade de Curitiba há registros da 
formulação de políticas públicas articulando promoção da saúde e atividade física com a reestruturação do espaço urbano, que resultaram em diversas ações e programas de sucesso como o Programa CuritibAtiva, iniciado em 1998, e que tem resultados positivos até hoje (HALLAL, 2009).

A partir de 2000, projetos com essa perspectiva foram se expandindo para várias cidades do País, como em Recife que, em 2002, desenvolveu uma proposta com base comunitária, articulando a Estratégia Saúde da Família (ESF) com a área de saúde mental, denominada Programa Academia da Cidade (PAC). Já em 2005 foi a vez de Maringá, com a implantação do Projeto Maringá Mais Saudável, uma experiência de incentivo a atividade física semelhante a que ocorre há mais de 15 anos nas praças das cidades da China, país com tradição na prática de atividades corporais ao ar livre como prevenção de doenças e promoção da saúde (BRASIL, 2011A).

Em 2006, com a criação da Política Nacional de Promoção da Saúde, os projetos de atividade física desenvolvidos no País foram alinhados às suas diretrizes, destacando-se aqui os projetos Pratique Saúde, no referido ano, e a campanha Time Mais Saúde, em 2008 (MALTA, 2009). Com o objetivo principal de fortalecer a promoção da saúde e a produção do cuidado e de modos de vida saudáveis, foi instituído em 2011 o Programa Academia da Saúde (PAS), por meio da Portaria Ministerial $\mathrm{n}^{\circ} 719$ (redefinida pela portaria $^{\circ}{ }^{2.681}$, de 7 de novembro de 2013), com o objetivo de contribuir para a promoção da saúde a partir da produção de modos de vida saudáveis por meio da implantação de polos com infraestrutura, equipamentos e profissionais qualificados.

O PAS é constituído por espaços públicos que têm por objetivos configurarem-se como pontos de atenção da rede de assistência à saúde e estabelecerem-se como espaços de ressignificação de conhecimentos. Seus princípios são a participação popular e coletiva, interdisciplinaridade, integralidade, intergeracionalidade e a territorialidade, tendo ainda como pressuposto ampliar o acesso da população às políticas de promoção da saúde e promover, junto às linhas de cuidado, atenção integral à população no território adscrito da ESF. A formulação do PAS é resultado de experiências exitosas de programas populacionais brasileiros de atividade física e, neste cenário, surgiram também as iniciativas de monitoramento e avaliação dessas intervenções, com destaque para o projeto de Avaliação de Efetividade de Programas de Atividade Física no Brasil (BRASIL, 2011A), conduzido pelo Ministério da Saúde em 2011.

No município do Rio de Janeiro, o PAS foi iniciado em 2009 com a denominação de Programa Academia Carioca da Saúde (Pacas) e em 2013 já estava presente em 172 Unidades Básicas de Saúde (UBS), incluindo as Clínicas da Família (CF) (RIO DE JANEIRO, 2013). O Pacas trabalha de forma similar ao PAS e tem como perspectivas o aumento da prática regular de atividade física e da efetividade das ações de promoção da saúde na Atenção Básica, além do fortalecimento de ações intersetoriais e a capilarização de conhecimentos sobre estilos de vida saudáveis, sendo aberto à participação de todos, com prioridade à modalidade aparelho para pessoas idosas, portadoras de hipertensão arterial e diabetes mellitus, e excesso de peso (FRAGA ET AL., 2013).

Face aos benefícios que a atividade física proporciona na população e à projeção de expansão das Academias Carioca da Saúde (Acas) no município do Rio de Janeiro, torna-se fundamental acompanhar a sua implementação e subsidiar a gestão local quanto a estruturação e consolidação dessa estratégia no campo da promoção da saúde. Inserido nesse contexto, este artigo tem como finalidade apresentar os resultados de um estudo de avaliabilidade do Programa Academia Carioca da Saúde - Escola Manguinhos, visando ampliar a compreensão sobre o desenho da intervenção e favorecer a 
utilização do processo avaliativo para melhoria do programa.

\section{Metodologia}

Trata-se de um estudo de avaliabilidade que é compreendido como um exame sistemático e preliminar, que antecede a avaliação de um programa, com a finalidade de identificar se o seu estágio de desenvolvimento permite sua avaliação, se os objetivos da intervenção respondem à situação-problema que o originou e quais aspectos do programa devem ser priorizados na avaliação (THURSTON; RAMALIU, 2005).

Optou-se por uma abordagem qualitativa com estudo de caso intrínseco, pois o mesmo trata de uma única realidade que poderá ser estudada profundamente, a fim de explicar o objeto de estudo (OLIVEIRA, 2012). A unidade de análise foi o Programa Academia Carioca da Saúde Escola Manguinhos. As etapas do plano de avaliabilidade realizadas nessa pesquisa foram as sugeridas por Leviton et al. (1998) e incluem: (a) análise documental para esclarecimento sobre os objetivos e metas do programa; (b) entrevista com os informantes-chave; (c) modelização da intervenção; (d) realização de oficina para pactuação do modelo lógico com os interessados; (e) formulação das perguntas avaliativas. A figura 1 apresenta um fluxograma utilizado para operacionalização do estudo.

Figura 1. Etapas utilizadas para delinear o estudo de avaliabilidade do Programa Academia Carioca da Saúde em 2013

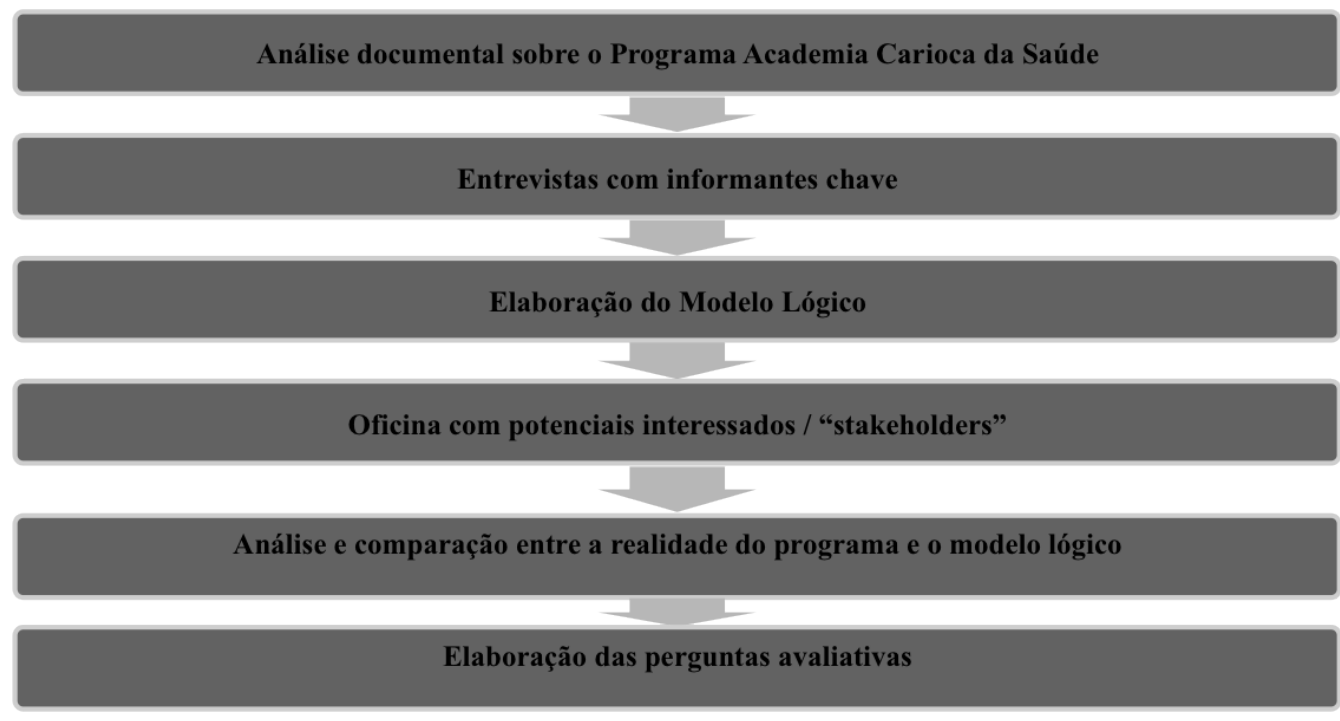

Fonte: Elaboração própria

Foram revisados os documentos publicados e/ou disponibilizados pelo Ministério da Saúde e pela Secretaria Municipal de Saúde do Rio de Janeiro sobre a temática, no período compreendido entre 2009 e 2012. Os critérios de inclusão adotados foram norteados pelos princípios básicos apontados por Richardson (1999): representatividade, adequação e exaustividade.

Para a entrevista face a face, o foco de interesse foi a opinião dos chamados stakeholders (atores interessados no processo avaliativo), 
e a amostra da pesquisa foi do tipo intencional e não probabilística (MARCONI; LAKATOS, 2011). $\mathrm{Na}$ presente pesquisa foram considerados informantes-chave: profissionais da educação física do Programa Academia Carioca da Saúde, gestores e profissionais das Equipes de Saúde da Família de Manguinhos.

As informações foram coletadas por meio de um roteiro de entrevistas semiestruturado, com dez perguntas relacionadas ao conhecimento sobre os objetivos e as ações desenvolvidas no programa, os insumos necessários, os contextos social, político e organizacional que permeiam a implementação dessa prática no território de Manguinhos, a importância do Programa Academia Carioca da Saúde para Promoção da Saúde e também sobre as ferramentas de monitoramento e avaliação do programa. Antecedendo à coleta de dados, foi entregue o Termo de Consentimento Livre e Esclarecido (TCLE) aos participantes, realçando o sigilo e confidencialidade.

Com base na análise documental e nas entrevistas com os informantes-chave, foi elaborado o Modelo Lógico do Programa Academia Carioca da Saúde - Escola Manguinhos, de forma a explicitar o funcionamento do programa e as etapas necessárias para transformação dos objetivos em metas, com base na proposição de Champanhe et al. (2011). Com o intuito de validá-lo, foi realizada uma oficina utilizando a técnica de consenso, com a perspectiva de auxiliar na produção de um resultado que seja de comum acordo entre os participantes do programa. Para esse processo foram convidados os informantes-chave que participaram da entrevista, além do coordenador municipal do programa e especialistas em avaliação de programas, que analisaram o modelo lógico de acordo com os critérios de clareza na descrição dos objetivos do programa, pertinência das dimensões e adequação dos insumos e ações/atividades para a operacionalização da intervenção. Os atores envolvidos validaram o conteúdo e a plausibilidade das relações estabelecidas no modelo lógico.
Esse projeto foi aprovado pelo Comitê de Ética em Pesquisa da Ensp/Fiocruz sob o parecer 177.223, no dia 20/12/2012, e CAAE: 10709012.8.0000.5240.

\section{Resultados e discussão}

\section{Cenário da intervenção}

O Complexo de Manguinhos é um bairro da zona norte do município do Rio de Janeiro, localizado na Área Programática (AP) 3.1 e formado por 13 comunidades. Com população de 44.051 habitantes, é uma localidade que se caracteriza por apresentar um dos piores Índices de Desenvolvimento Humano (IDH) da cidade, situando-se em $122^{\circ}$ lugar dentre os 126 bairros (CASANOVA; TEIXEIRA; ENGSTROM, 2014). A ESF foi implantada em 2000, porém a maior expansão ocorreu em 2010 com a implantação da Clínica da Família, alcançando uma cobertura de $100 \%$ da população residente por meio da atuação de 13 equipes. Cada equipe é composta por 01 médico, 01 enfermeiro, 01 técnico de enfermagem, 06 agentes comunitários de saúde e 01 agente de vigilância em saúde, além de 01 Equipe de Consultório na Rua, 01 Núcleo de Apoio à Saúde da Família (Nasf), 01 Equipe de Atenção Domiciliar e uma Acas, objeto deste estudo (BRASIL, 2014).

A Acas está inserida no âmbito do Território Integrado de Atenção à Saúde (Teias), parceria entre a Secretaria Municipal de Saúde do Rio de Janeiro e a Escola Nacional de Saúde Pública/Fiocruz. O Teias - Escola Manguinhos orienta-se pelo reconhecimento do território e da comunidade como atores agindo articuladamente, para a compreensão dos determinantes sociais da saúde a partir da realidade local. Nesse sentido, organiza-se para produzir novos conhecimentos aplicáveis a diversas realidades e necessidades do território, visando sempre ampliar a participação comunitária e intersetorial, com coleta de dados sistemáticos 
que permitam apoiar a gestão na tomada de decisão. Sendo o Pacas/Escola a única Acas inserida em um Território Escola no município do Rio de Janeiro, foi definida a utilização da terminologia 'Programa Academia Carioca da Saúde - Escola Manguinhos' (ENGSTROM; FONSECA; LEIMANN, 2012).

\section{Análise documental e entrevistas}

A análise documental permitiu identificar um conjunto de informações coerentes e consistentes para delimitar os objetivos e metas do Programa Academia Carioca da Saúde - Escola Manguinhos, que são: fortalecer as práticas interdisciplinares no território com foco nas ações de promoção da saúde, aumentar a adesão da população à prática de atividade física regular, contribuir para melhorar o acesso da comunidade às informações sobre hábitos e comportamentos mais saudáveis e estimular o vínculo com a mesma, fortalecendo a participação social.

Cabe mencionar que o Pacas, no município do Rio de Janeiro, passou por algumas reformulações desdeasuaimplantação. O programa foi implementado em 2009, por meio de uma Resolução Conjunta da Secretaria Especial de Envelhecimento Saudável e Qualidade de Vida com a Secretaria Municipal de Saúde e Defesa Civil - Resolução ${ }^{0}$ 002/2009 -, assim como os Projetos Academia da Terceira Idade e Academia Carioca da Saúde e Envelhecimento Saudável. O primeiro caracterizava-se por um projeto com instalações em logradouros públicos e o segundo por unidades básicas de saúde, porém ambos tinham o propósito de conscientização dos idosos sobre a importância da prática regular de exercícios físicos, no sentido de melhorar sua qualidade de vida e prevenir doenças e agravos não transmissíveis. O programa Envelhecimento Saudável foi rebatizado de Academia Carioca da Saúde (Acas) e hoje faz parte da Superintendência de Promoção da Saúde da Secretaria Municipal de Saúde, sob a coordenação da Assessoria de Atividade
Física. Cotejando-o com a proposta do Programa Academia da Saúde, de âmbito ministerial, percebem-se similaridades em relação à proposta de estímulo à prática de atividade e promoção da saúde, vinculado a uma unidade da ESF, porém existem diferenças quanto ao formato dos aparelhos utilizados.

A etapa de entrevistas com atores interessados no programa revelou aspectos importantes relacionados à organização do Programa Academia Carioca da Saúde Escola Manguinhos no território, bem como sobre os fatores contextuais que permearam a sua implementação.

Trata-se de um programa público vinculado à Secretaria Municipal de Saúde do Rio de Janeiro e o usuário, para participar, pode ser inserido a partir de uma demanda espontânea ou através de referência da Clínica da Família. Na demanda espontânea, o usuário procura voluntariamente o educador físico do Pacas e manifesta seu interesse em participar das atividades do programa e, no caso do encaminhamento, passa por uma avaliação para ser direcionado ao protocolo de atividades desenvolvidas. Em ambas as situações é realizada a anamnese que, além de buscar identificar informações socioeconômicas e de qualidade de vida do usuário (SF-36 - instrumento genérico que abrange alguns componentes, como capacidade funcional e estado geral), analisa a prontidão dele para a prática de atividade física, assim como a entrada dos dados no sistema de prontuário eletrônico. Com relação ao contexto, os fatores facilitadores relacionados ao Pacas citados pelos entrevistados foram: o fato da academia ter sido uma solicitação da comunidade, o aumento de vínculo entre a população da área e as Equipes de Saúde da Família - o que ajudou a traçar melhor as necessidades de saúde do território - e o incentivo à busca ativa de usuários faltosos na unidade de saúde. Ademais, observou-se nas entrevistas que essa estratégia fortaleceu o apoio institucional e induziu maior autonomia para a equipe planejar o seu processo de 
trabalho. Também foram referidas possíveis barreiras para a consolidação do programa: a violência no território, a forma de contratação dos profissionais, a dificuldade de manutenção dos equipamentos do módulo da academia, a insistência de uma cultura de medicalização e a qualificação de alguns profissionais envolvidos no programa.

Entender como se dá a relação com o ambiente político e social (contexto externo) em que o programa se insere e o ambiente organizacional (contexto institucional) é importante para refletir sobre as potencialidades, bem como sobre os aspectos que podem interferir negativamente nas condições de implantação da intervenção e nos processos de produção dos efeitos. Ao analisar os determinantes contextuais do Pacas em Manguinhos, é possível compreender que existem condições do meio que tornam plausível sua implantação integral. No entanto, é preciso ficar atento às barreiras existentes, principalmente aquelas relacionadas às questões estruturais da sociedade e, portanto, mais complexas em sua resolubilidade, como a violência, por exemplo.

\section{Modelização da intervenção e formulação das perguntas avaliativas}

A construção do modelo lógico do Programa Academia Carioca da Saúde - Escola Manguinhos ajudou a identificar as relações entre os insumos, as atividades do programa e os efeitos esperados com a perspectiva de potencializar a estratégia no âmbito da promoção da saúde. A identificação dos componentes estruturais baseou-se nos objetivos do programa, e as hipóteses são de que os recursos certos serão transformados em ações necessárias para os beneficiários, e isso, em um contexto favorável, irá levar aos resultados que o programa pretende alcançar (CHEN, 1990).

No campo da promoção da saúde, diversas pesquisas foram conduzidas com o intuito de realizar a modelização da intervenção e, entre essas, cabe mencionar um estudo do Ministério da Saúde em parceria com diversas universidades brasileiras, o Center For Disease Control (CDC) norte-americano e o Projeto Guia, que apontou a importância do modelo lógico para definir o que deve ser avaliado, na medida em que a representação visual expressa a forma de implementação do programa para atingir os objetivos propostos (BRASIL, 2011A).

A estrutura e funcionamento do Programa Academia Carioca da Saúde - Escola Manguinhos, bem como os resultados esperados, podem ser visualizados de maneira mais clara e objetiva no modelo lógico do programa apresentado na figura 2, onde se destacam os seguintes componentes: 1) Articulação Intersetorial; 2) Assistência; 3) Ensino/Formação; 4) Gestão. 


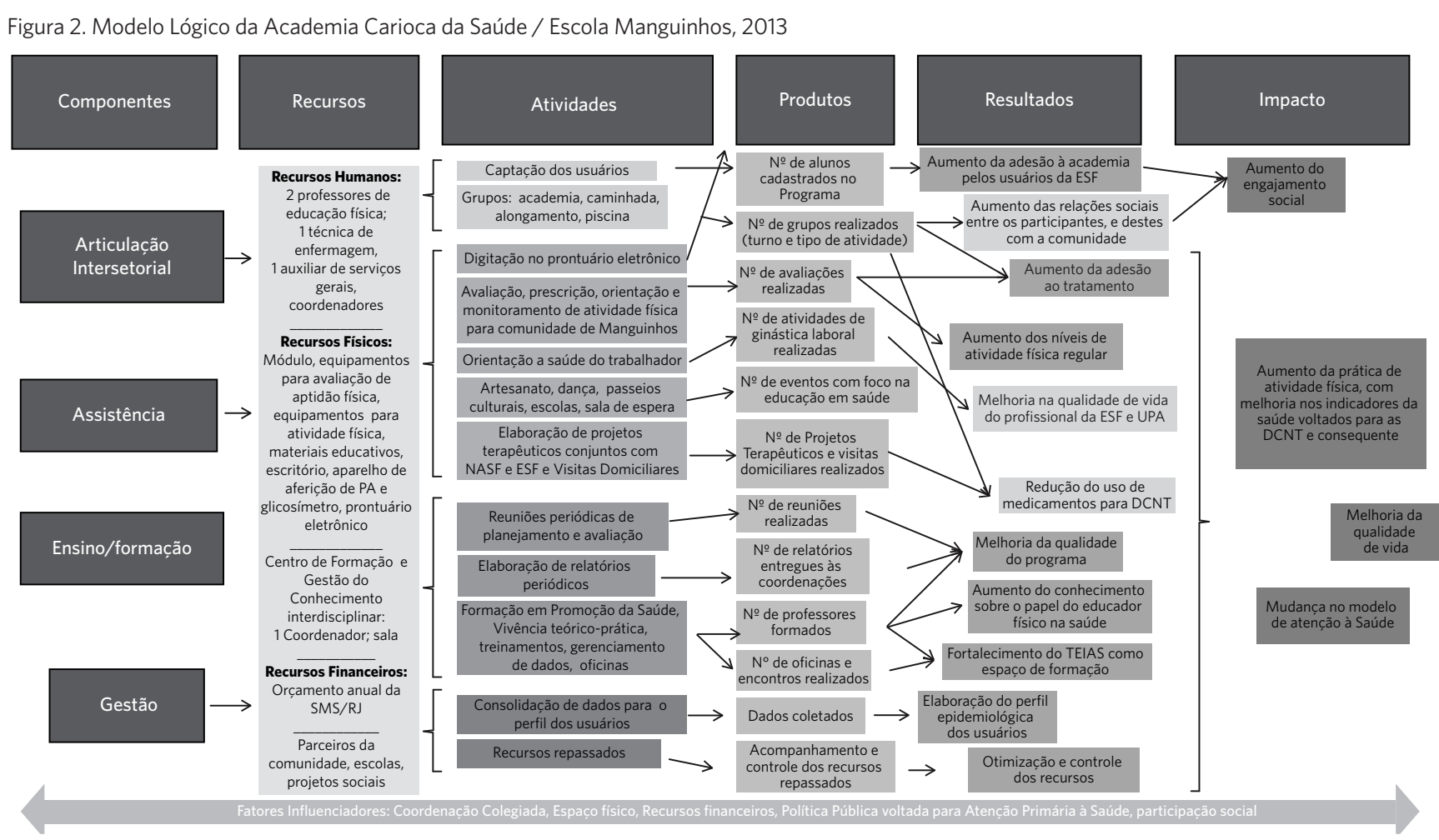

No componente Articulação Intersetorial, observou-se que as atividades têm enfoque mais amplo do que somente o incentivo à prática de atividade física. Incluem, por exemplo, a articulação com o Programa Saúde na Escola (PSE) e com os dispositivos institucionais de gestão participativa, que se reúnem para pensar a complexa questão da saúde em Manguinhos: Colegiados das Unidades de Saúde, Conselho Gestor Intersetorial, Seminário Temáticos de Saúde, Conferências Territoriais de Saúde, Ouvidoria e Ferramentas de Comunicação e espaços de participação social (ENGSTROM; FONSECA; LEIMANN, 2012).

Trata-se, portanto, de um componente fundamental para o desenvolvimento de ações de promoção da saúde, pois envolve atividades que requerem um movimento mais complexo e abrangente, englobando diferentes setores da sociedade que se corresponsabilizam pela garantia da saúde como direito fundamental do cidadão. É fundamental reconhecer que o Programa Academia Carioca da Saúde - Escola Manguinhos pode ganhar maior amplitude a partir de interlocuções da política, da gestão, das práticas assistenciais e das relações com a população, com enfoque interinstitucional e intersetorial. Com relação ao componente Assistência, foram identificadas atividades próprias do Pacas, tais como: atendimentos individuais para avaliação física; atendimento diário para aferição da pressão arterial, antes e após o exercício; grupos de caminhada e alongamento na comunidade; atividades de ginástica, alongamento e dança; atividades na piscina (ou aquáticas), como hidroginástica e natação; grupos como de controle do tabagismo; passeios culturais; além das atividades de ginástica laboral e dicas ergonômicas para o ambiente de trabalho da CF. Foram também incluídas as ações relacionadas ao Nasf, com destaque para discussão de 
caso dos Projetos Terapêuticos Singulares (PTS) com ênfase nas ações de promoção da saúde.

Esse cenário reforça a intenção da implementação do Pacas no território, que é oferecer atividades físicas suficientes para que seus usuários se aproximem ou alcancem as recomendações mínimas da prática regular de atividade física sugerida para a manutenção de bons níveis de saúde, além de estimular a realização de algumas atividades de maneira autônoma, principalmente nos dias em que os profissionais não desenvolvem suas programações em Manguinhos. No entanto, para a sustentabilidade dessa prática é de fundamental importância que haja um ambiente favorável à adoção de um estilo de vida mais ativo, o que pressupõe, além da disponibilidade dos equipamentos, a presença de praças, parques públicos, pavimentação de calçadas, pistas de caminhada e ciclovias, bem como dar prioridade à questão da segurança, o que evidencia a necessidade do envolvimento de vários setores da sociedade (SAELENS, ET AL., 2003).

Quanto ao componente Ensino/Formação, existem atividades de apoio pedagógico aos educadores físicos da rede do Pacas, desenvolvidas por meio do Centro de Formação e Gestão do Conhecimento Interdisciplinar, que conta com um professor/coordenador e ocorre tanto nos espaços físicos internos da Clínica de Família quanto no espaço do próprio Programa Academia Carioca da Saúde - Escola Manguinhos. As atividades são voltadas para o treinamento de novos profissionais e a educação continuada, e ainda contam com reuniões periódicas para formação em promoção da saúde com foco no programa. De fato, trata-se de uma estratégia importante, tendo em vista a necessidade de constituir um espaço de reflexão coletiva sobre a liberdade de escolha dos indivíduos, no âmbito da promoção da saúde, o que inclui a prática de atividade física. Assim, a educação se integra e se articula no intuito de promover a produção de conhecimentos norteados pela necessidade de transformações na vida das pessoas e, consequentemente, na realidade de uma sociedade.

No Programa Academia Carioca da Saúde - Escola Manguinhos, o componente Gestão é composto por atividades com foco no gerenciamento e monitoramento do programa. Como a consolidação dos dados dos usuários é registrada e arquivada em um banco de dados, essas informações são discutidas com a coordenação, profissionais e gestores, permitindo a reorientação das ações do programa com o olhar na integralidade da assistência prestada ao usuário, à família e à comunidade. Trata-se também de um registro da produção dos profissionais de saúde no desenvolvimento de ações do Pacas em Manguinhos. Cabe ressaltar que o monitoramento do programa ocorre no âmbito do planejamento, por meio de instrumentos de gestão e indicadores específicos, os quais servirão de referência para avaliar, em longo prazo, a efetividade do programa na população.

Após a construção da primeira versão do modelo lógico, este foi pactuado em uma oficina de validação, com o uso da técnica de consenso, a fim de verificar a consistência lógica interna do programa, auxiliando na produção de um resultado que seja de comum acordo dos participantes. Devemos considerar que são estes atores - as organizações que possuem relação direta com o programa - assim como os atores que financiam e também apoiam tecnicamente, que podem ser positiva ou negativamente afetados, sendo capazes de influenciar a sua sustentabilidade (JOPPERT; SILVA, 2012).

A apresentação do modelo lógico permitiu acordar entre os interessados aquilo que deve ser mensurado e qual a parcela de contribuição do programa nos resultados esperados, facilitando o diálogo sobre o entendimento dos interesses de cada um (NOAA, 2010). Mesmo com as limitações "o desenho do modelo lógico possibilita aos gestores e avaliadores uma visão mais clara acerca da 
racionalidade da construção da intervenção e das relações causais empregadas na sua elaboração" (BEZERRA; CAZARIN; ALVES, 2010, P. 77).

Com efeito, o modelo lógico final mostrou o percurso do Programa Academia Carioca da Saúde - Escola Manguinhos para que a população de Manguinhos seja mais ativa e estimulou a coordenação a procurar formas de fortalecer o papel da estratégia no âmbito da política de promoção da saúde. Identificouse a necessidade de incentivar as parcerias interinstitucionais e promover maior investimento em cursos que possam capacitar os profissionais na discussão da promoção da saúde, visando aumentar a potencialidade do programa, principalmente no que tange a participação comunitária. Outro ponto que merece destaque é o investimento na construção de alianças com outras áreas técnicas que permitam ampliar o escopo das atividades de promoção da saúde, principalmente com áreas como de alimentação e nutrição e controle do tabagismo, visando fortalecer a sustentabilidade do programa no território em médio e longo prazos.

Por fim, a validação permitiu ter mais clareza sobre as perguntas avaliativas necessárias para um estudo avaliativo mais sistemático, colaborando com um realinhamento estratégico e contínuo das ações. A partir do modelo lógico, foram identificadas algumas perguntas avaliativas, tais como: Qual o grau de implantação do programa Academia Carioca da Saúde no território de Manguinhos? As atividades são realizadas de acordo com o preconizado? Qual o grau de adesão da população ao Programa Academia Carioca da Saúde? Quais facilitadores e barreiras foram percebidos no processo de implementação? Além disso, outros desenhos de estudos avaliativos serão necessários para responder às perguntas avaliativas com efeitos mais finalísticos, tais como: A estratégia de promoção da saúde no território é custo-efetivo? Qual o impacto das ações do Programa Academia Carioca da Saúde no território de Manguinhos?

\section{Considerações finais}

A análise de avaliabilidade empreendida no estudo permitiu evidenciar a adequação do desenho do Programa Academia Carioca da Saúde - Escola Manguinhos, uma vez que implementa as diretrizes da Política de Promoção da Saúde nos seguintes aspectos: ampliação do acesso regular às práticas de atividade física e corporais; estímulo à criação de grupos de educação em saúde que promovam modos de vida saudáveis; estímulo à participação comunitária, rodas de convívio social; integração com a rede intersetorial de serviços de apoio no território, com foco não só no controle das doenças crônicas não transmissíveis, como também na melhoria da qualidade de vida da população.

Embora ainda em fase de consolidação, o que não permitiria empreender uma avaliação dos seus resultados em longo prazo, é possível e recomendável a realização de estudos mais sistemáticos sobre o processo de implementação do Pacas no território de Manguinhos, como por exemplo, quanto ao grau em que as atividades previstas estão sendo implementadas com os recursos previstos, em quantidade e com a qualidade esperada; quanto à cobertura e acesso, à satisfação dos usuários, à inserção na Política de Promoção da Saúde no município do Rio de Janeiro e ao diálogo com outras políticas, como o Plano de Ações Estratégicas para o Enfrentamento das Doenças Crônicas Não Transmissíveis .

Esse processo avaliativo poderá ser útil para analisar os diferentes fatores em jogo na dinâmica interna do programa e como interagem para facilitar ou bloquear o alcance dos objetivos pretendidos. Recomenda-se que, a partir deste trabalho, seja feito um investimento maior na avaliação do programa, com o uso das perguntas avaliativas mencionadas na pesquisa, de forma a buscar uma intervenção de qualidade, eficiente e efetiva, para a melhoria do padrão de cuidado prestado à população. 


\section{Referências}

BEZERRA, L. C. A; CAZARIN, G.; ALVES, C. K. A. Modelagem de programas: da teoria à operacionalização. In: SAMICO, I. et al. (Org). Avaliação em Saúde: bases conceituais e Operacionais. Rio de Janeiro: MedBook, 2010.

BRASIL. Ministério da Saúde. Secretaria de Vigilância em Saúde. Avaliação de Efetividade de Programas de Atividade Física no Brasil. Brasília, DF: Ministério da Saúde, 2011a.

Ministério da Saúde. Secretaria de Vigilância em Saúde. Departamento de Análise de Situação de Saúde. Plano de Ações Estratégicas para o Enfrentamento das Doenças Crônicas Não Transmissíveis (DCNT) no Brasil 2011-2022. Brasília, DF: Ministério da Saúde, 2011b.

CASANOVA, A. O.; TEIXEIRA, M. B.; ENGSTRON, E. M. O apoio institucional como pilar na co-gestão da atenção primária à saúde: a experiência do Programa Teias-Escola Manguinhos no Rio de Janeiro. Revista Ciência e Saúde Coletiva. Rio de Janeiro, v. 19, n. 11, p. 4417-4426, 2014. Disponível em: <http://dx.doi. org/10.1590/1413-812320141911.14702013>. Acesso em: 1 dez. 2014.

CHAMPAGNE, F. et al. Análise Estratégica. In: HARTZ, Z. et al. (Org.) Avaliação: Conceitos e métodos. Rio de Janeiro: Editora Fiocruz, 2011, p. 95-104.

CHEN, H. T. Theory-Driven Evaluations. Newbury Park: Sage, 1990.

ENGSTROM, E. FONSECA, Z.; LEIMANN, B. A experiência do território Escola Manguinhos na Atenção Primária de Saúde. Rio de Janeiro: Editora Fiocruz, 2012.

FERREIRA, M. S., CASTIEL, L. D.; CARDOSO, M. H. C. A. Atividade física na perspectiva da nova promoção da saúde: contradições de um programa institucional. Ciência \&t Saúde Coletiva, Rio de janeiro, v. 16, Supl. 1, p. 865-872, 2011.

FRAGA, A. B. et al. (Org). Curso de extensão em promoção da saúde para gestores do SUS com enfoque no programa academia da saúde. Brasília, DF: Ministério da Saúde, 2013, 144 p.

HALLAL, P. C. Avaliação de programas comunitários de promoção da atividade física: o caso de Curitiba,
Paraná. Revista Brasileira de Atividade Física \&t Saúde, Pelotas, v. 14, n. 2, 2009.

JOPPERT, M. P.; SILVA, R. R. Guia Metodológico para Monitoramento e Avaliação Participativa de Ações Municipais. Brasília, DF: CNM: Pnud, 2012.

KOHL, H. W.; The pandemic of physical inactivity: global action for public health. The Lancet, London, v. 380, p. 294 - 305, Jul. 2012. Disponível em: <http:// www.thelancet.com/pdfs/journals/lancet/PIISO1406736(12)60898-8.pdf>. Acesso em: 4 ago. 2012.

LEVITON, L. C. et al. Teaching evaluation using evaluability assessment. Evaluation. London, n. 4, p. 389 409, 1998.

MALTA, D. C. et al. A Política Nacional de Promoção da Saúde e a agenda da atividade física no contexto do SUS. Epidemiol. Serv. Saúde, Brasília, DF, v. 18, n. 1, p. 79-86, 2009.

MARCONI, M. A.; LAKATOS, E. M. Técnicas de pesqui$s a$ : Planejamento e execução de pesquisa, amostragens e técnicas de pesquisa, elaboração, análise e interpretação de dados. 7 ed. São Paulo: Atlas, 2011. 277 p.

\section{NATIONAL OCEANIC AND ATMOSPHERIC} ADMINISTRATION (NOAA). Introduction to Planning and Facilitating Effective Meeting. 2010. Disponível em: <https://coast.noaa.gov/digitalcoast/ sites/default/files/files/1366310745/planning_and_facilitating_effective_meetings.pdf?redirect $=3010 \mathrm{~cm}>$. Acesso em: 7 jan. 2015.

OLIVEIRA, M. M. Como fazer pesquisa qualitativa. Petrópolis: Vozes, 2012. 232 p.

RIBEIRO, A. G.; COTTA, R. M. M.; RIBEIRO, S. M. R. A promoção da saúde e a prevenção integrada dos fatores de risco para doenças cardiovasculares. Ciência \&t Saúde Coletiva, Rio de Janeiro, v. 17, n. 1, Jan. 2012. Disponível em: < http://www. scielo.br/scielo.php?script=sci_arttext\&pid=S1413$-81232012000100002 \& \operatorname{lng}=e n \& n r m=$ iso $>$. Acesso em: 17 jan. 2015.

RICHARDSON, R. J. Pesquisa social: métodos e técnicas. São Paulo: Atlas, 1999. 
RIO DE JANEIRO. Secretaria Municipal de Saúde e Defesa Civil. Subsecretaria de Atenção Primária, Vigilância e Promoção de Saúde. Superintendência de Atenção Primária. A Reforma da Atenção Primária no Rio de Janeiro 2009-2012. Rio de Janeiro: SMSDC, 2013. 52 p.

SAELENS, B. E. et al. Neighborhood-Based Differences in Physical Activity: Na environment Scale Evaluation. Research and Practice. American Journal of Public Health, v. 93, n. 9, Sep. 2003. Disponível em: <http:// www.ncbi.nlm.nih.gov/pmc/articles/PMC1448009/ pdf/0931552.pdf>. Acesso em: 2 abr. 2013.

SCHMIDT, M. I. et al. Doenças Crônicas não transmissíveis no Brasil: carga e desafios atuais. Saúde no Brasil. Maio, 2011, p. 65-74. Disponível em: <http://www.thelancet.com/series/health-in-brazil>. Acesso em: 25 jan. 2013.
THURSTON, W. E.; RAMALIU, A. Evaluability Assessment of survivors of a torture program: Lessons learned. The Canadian Journal of Program Evaluation, Renfrew, v. 20, n. 2, p. 1-25, 2005.

World Health Organization (WHO). Global health risks: mortality and burden of disease attributable to selected major risks. Genebra, 2009. Disponível em: <http://www.who.int/healthinfo/global_burden_disease/GlobalHealthRisks_report_full.pdf>. Acesso em: 7 jan. 2013.

Recebido para publicação em abril de 2014

Versão final em janeiro de 2015

Conflito de interesse: inexistente

Suporte financeiro: não houve 